\title{
Pelvic Exenteration for Locally Advanced and Relapsed Pelvic Malignancies - An Analysis of 100 Cases
}

\author{
NICOLAE BACALBASA ${ }^{1,2,3^{*}}$, IRINA BALESCU ${ }^{4}$, MIHAELA VILCU $^{1,2}$, \\ ADRIAN NEACSU ${ }^{1}$, SIMONA DIMA $^{3}$, ADINA CROITORU ${ }^{3,5^{*}}$ and IULIAN BREZEAN ${ }^{1,2}$ \\ 1 "Carol Davila" University of Medicine and Pharmacy, Bucharest, Romania; \\ 2 "I. Cantacuzino" Clinical Hospital, Bucharest, Romania; \\ 3 "Fundeni" Clinical Institute - Center of Excellence in Translational Medicine, Bucharest, Romania; \\ 4 "Ponderas" Academic Hospital, Bucharest, Romania; \\ 5 "Titu Maiorescu” University, Bucharest, Romania
}

\begin{abstract}
Background/Aim: Although pelvic exenteration is an aggressive surgical procedure, it remains almost the only curative solution for patients diagnosed with large pelvic malignancies. Patients and Methods: We present a series of 100 patients submitted to pelvic exenteration with curative intent. Results: The origin of the primary tumor was most commonly represented by cervical cancer, followed by, endometrial cancer, rectal cancer, ovarian cancer and vulvovaginal cancer. An R0 resection was confirmed in 68 cases, while the remaining 32 cases presented lateral positive resection margins or perineal positive margins. The postoperative morbidity rate was $37 \%$ while the mortality rate was $3 \%$. As for the-long term outcomes, the median overall survival time was 38.7 months, being most significantly influenced by the origin of the primary tumor. Conclusion: Although pelvic exenteration is still associated with an increased morbidity, an important improvement in the long-term survival can be achieved, especially if radical resection is feasible.
\end{abstract}

Reported for the first time in 1948 in order to alleviate the symptoms of women diagnosed with advanced or relapsed gynecological malignancies and described at that moment as "the most radical surgical attack so far described for pelvic cancer", pelvic exenteration became in the last decades the

This article is freely accessible online.

*These Authors contributed equally to this study.

Correspondence to: Irina Balescu, "Ponderas" Academic Hospital, Bucharest, Romania. Tel: +40 724077709, e-mail: irina.balescu@ ponderas-ah.ro

Key Words: Pelvic exenteration, curative, survival. option of choice in treating such cases with radical intent (1). Although initially the long-term outcomes were rather poor due to the local extension of the tumor, an important benefit of survival has been observed, especially in cases in which a radical surgical procedure and negative resection margins could be achieved (2). Moreover, since the first studies regarding the notions of ultra-radical compartmentalized resection, the process of local invasion and relapse of such tumors has been better understood and, in consequence, a more specific and personalized surgical treatment has been proposed $(3,4)$. According to Hockel's studies, the local invasion of the tumor initially respects the embryological and anatomical borders of the gynecological, digestive and urological tract. Once these boundaries are destroyed, the tumor will involve multiple anatomical compartments. In such cases all these compartments, as well as all the structures having the same embryological origin should be resected in order to excise all the microscopical tumoral foci. However, the procedure is still associated with significant rates of postoperative complications which might reach up to $50 \%$ in specialized centers and even with early postoperative mortality (3). In the meantime, the quality of life might be significantly impeded especially in cases in which a reconstructive phase is not feasible. The current paper presents our experience with this procedure over an interval of three years, in which 100 pelvic exenterations were performed and aimed to evaluate the early postoperative evolution, as well as the prognostic factors for long-term outcomes.

\section{Patients and Methods}

Data of patients submitted to surgery for locally advanced or relapsed pelvic tumors between January 2014 and December 2017 were retrospectively reviewed. Among the 133 patients identified, pelvic exenteration was feasible in 100 cases; in the other 33 cases the procedure was aborted due to the presence of distant metastases which had not been detected during the preoperative work-up or due 
to the local extent of the disease involving unresectable structures. The extent of the disease was ruled out after performing a chest and abdominal computed tomography, while the local extension of the tumor was investigated by magnetic resonance imaging (MRI). All cases were initially discussed in a tumor board in the presence of a medical oncologist, surgical oncologist, oncological surgeon, radiotherapist, oncologist and urologist. Patients in whom a neoadjuvant chemo-irradiation protocol was applied were rediscussed during the multidisciplinary meetings after ending the neoadjuvant treatment. Demographic data as well as intraoperative and postoperative details were retrospectively reviewed. The presence of postoperative complications was assessed according to Dindo-Clavien scale (5). After identifying the cases eligible for this study the approval of the ethics committee was obtained (no. $32 / 2019$ ). The obtained results were considered as statistically significant if a $p$-value below 0.05 was obtained.

\section{Results}

The median age at the time of conducting the study was 47 years (range $=38-76$ years), the median body mass index $(\mathrm{BMI})$ value was $28,5 \mathrm{~kg} / \mathrm{m}^{2}$ (range $=18-36 \mathrm{~kg} / \mathrm{m}^{2}$ ), while the gender distribution female/male was $91 / 9$. The origin of the primary tumor was represented by cervical cancer (58 cases), endometrial cancer (13 cases), rectal cancer (12 cases), ovarian cancer (11 cases), vulvar cancer ( 3 cases) and vaginal cancer (3 cases). When it comes to the type of tumor (locally advanced or relapsed tumors), there were 55 cases submitted to pelvic exenteration for locally advanced disease, while the other 45 cases were submitted to surgery for pelvic relapse. In regard to cases submitted to pelvic exenteration for relapsed disease, surgery was performed after a median interval of nine months after ending the last therapeutic approach (which usually consisted of adjuvant chemotherapy). The histopathological studies demonstrated the presence of squamous cell carcinoma in 62 cases (56 cases with cervical cancer, all three cases diagnosed with vulvar cancer and all three cases with vaginal cancer), followed by adenocarcinoma in 36 cases ( 13 cases with endometrial cancer, 12 cases with rectal cancer and 11 cases with ovarian cancer) and neuroendocrine carcinoma in two cases (with cervical cancer). As for the degree of differentiation, there were 12 well differentiated tumors, 43 moderately differentiated tumors and 45 poorly differentiated tumors. Detailed data are shown in Table I.

Among the 55 patients submitted to pelvic exenteration for locally advanced tumors 48 cases had been previously submitted to radiotherapy, and in 23 cases neoadjuvant chemotherapy was also added. In the meantime, among the 45 cases submitted to pelvic exenteration for relapsed disease there were 12 cases which had been also submitted to radiotherapy as part of the initial treatment, eight of whom also associated with concurrent chemotherapy. When it comes to the type of surgery performed in cases diagnosed with recurrent tumors, it consisted of total radical hysterectomy with bilateral adnexectomy for cervical cancer in 31 cases, rectosigmoidian resection with colorectal anastomosis in six cases diagnosed with rectal cancer and total hysterectomy with bilateral adnexectomy for ovarian/endometrial cancer - in the remaining four cases.

As for the type of exenteration, a total supralevator exenteration was needed in 45 cases, an anterior exenteration was needed in 23 cases, a posterior exenteration was needed in 21 cases, a total infralevator exenteration was performed in six cases, while in the remaining five cases a laterally extended total pelvic exenteration was the option of choice. Intraoperative and early postoperative details are shown in Table II. Total supralevator or posterior exenteration reconstruction of the digestive tract by a colorectal anastomosis was the option of choice in 24 cases and was performed manually in 20 cases and mechanically in the remaining four cases. As for the other patients, we decided to perform a left-sided terminal colostomy. The main reasons which impeded us to perform an anastomosis were related to the heavy history of irradiation ( 23 cases), poor vascular supply of the anastomotic partners (20 cases) and poor nutritional status or obesity in the remaining nine cases. Ureteral reconstructions consisted of creating an ileal neobladder in 18 cases, Bricker reconstruction in 34 cases and cutaneous terminal urostomy in the remaining 25 cases. Ileal neobladder was the option of choice in young patients, with no previous history of irradiation in order to minimise the risk of postoperative complications. Perineal reconstructions were performed in two cases, both patients being submitted to surgery for locally advanced vulvar cancer and consisted of creating a gracilis myocutaneous flap.

There was no significant difference in terms of length of surgery or estimated blood loss between patients submitted to surgery for locally advanced or relapsed tumors; the median length of surgery was of 410 minutes for locally advanced tumors and 480 minutes for relapsed lesions $p=0.543$ ) while the estimated blood loss was $770 \mathrm{ml}$ for primary lesions and $890 \mathrm{ml}$ for relapsed tumors $(p=0.08)$. There was no statistically significant difference in regard to the necessity of transfusion administration.

During the early postoperative period (the first month postoperatively) there were 22 cases who developed serious complications (classified as Dindo-Clavien 3 or 4 complications) which consisted of pelvic abscesses in 16 cases, pulmonary embolism in three cases, and postoperative hemoperitoneum in eight cases. Three out of the 16 cases who developed a pelvic abscess were successfully treated in a conservative manner, while all the other cases required reoperation. Three of the 100 patients died during the postoperative period due to sepsis shock or peritonitis due to digestive or urinary tract leaks - one in each case, and by pulmonary embolism in association with bronchopneumonia in the remaining case.

The microscopic completeness of the resection was demonstrated by the histopathological analysis of the 
Table I. Demographic aspects and tumoral characteristics of the patients included in the present study.

\begin{tabular}{|c|c|}
\hline Characteristics & Value \\
\hline Median age (years) & 47 years $($ range= $=38-76$ years $)$ \\
\hline Median BMI $\left(\mathrm{kg} / \mathrm{m}^{2}\right)$ & $28.5 \mathrm{~kg} / \mathrm{m}^{2}\left(\right.$ range $\left.=18-36 \mathrm{~kg} / \mathrm{m}^{2}\right)$ \\
\hline \multicolumn{2}{|l|}{ Gender } \\
\hline Female & 91 \\
\hline Male & 9 \\
\hline \multicolumn{2}{|l|}{ Origin of the tumor } \\
\hline Cervical cancer & 58 \\
\hline Endometrial cancer & 13 \\
\hline Rectal cancer & 12 \\
\hline Ovarian cancer & 11 \\
\hline Vulvar cancer & 3 \\
\hline Vaginal cancer & 3 \\
\hline \multicolumn{2}{|l|}{ Type of tumor } \\
\hline Primary & 55 \\
\hline Relapsed & 45 \\
\hline \multicolumn{2}{|l|}{ Histological type } \\
\hline Squamous cell carcinoma & 62 \\
\hline Adenocarcinoma & 36 \\
\hline Neuroendocrine carcinoma & 2 \\
\hline \multicolumn{2}{|l|}{ Degree of differentiation } \\
\hline Well differentiated & 12 \\
\hline Moderately differentiated & 43 \\
\hline Poorly differentiated & 45 \\
\hline \multicolumn{2}{|l|}{ Associated comorbidities } \\
\hline Arterial hypertension & 34 \\
\hline Diabetes mellitus & 12 \\
\hline Chronic renal disease & 5 \\
\hline Chronic pulmonary disease & 2 \\
\hline \multicolumn{2}{|l|}{ History of prior irradiation } \\
\hline Locally advanced tumors & 48 \\
\hline Recurrent tumors & 12 \\
\hline
\end{tabular}

specimens in 68 cases. In the remaining 32 cases positive resection margins were encountered. Most often, the positivity of the margins was encountered on the lateral border of the specimen (in 28 cases) followed by the perineal area of resected skin (in four cases). Not surprisingly, positive resection margins were more often encountered when treating recurrent tumors originating from cervical cancer (in 18 cases) and rectal cancer (11 cases). The remaining three cases, in which an $\mathrm{R} 1$ resection was detected, had been submitted to surgery for primary tumors located at the level of the vulvar region (in two cases) and rectal cancer (in one case). When it comes to the dimensions of the resected tumors, $63 \%$ of cases presented larger than 5 $\mathrm{cm}$ lesions. In this category 45 of the 63 tumors were represented by primary lesions while the remaining 18 cases were recurrent lesions. As for the smaller lesions, 12 of the 37 cases were submitted to surgery for relapsed disease.

Histopathological details of the resected specimens are presented in Table III.
Table II. Intraoperative details and early outcomes after pelvic exenteration.

\begin{tabular}{|c|c|}
\hline Characteristic & Value \\
\hline \multicolumn{2}{|l|}{ Type of exenteration } \\
\hline Total supralevator & 45 \\
\hline Total infralevator & 6 \\
\hline Anterior & 23 \\
\hline Posterior & 21 \\
\hline Laterally extended & 5 \\
\hline \multicolumn{2}{|l|}{ Association of lymph node dissection } \\
\hline Bilateral pelvic lymph node dissection & 68 \\
\hline Paraaortic lymph node dissection & 31 \\
\hline Bilateral inguinal lymph node dissection & 6 \\
\hline \multicolumn{2}{|l|}{$\begin{array}{l}\text { Association of digestive tract } \\
\text { reconstructive procedures }\end{array}$} \\
\hline Colorectal anastomosis & 24 \\
\hline Terminal colostomy & 52 \\
\hline \multicolumn{2}{|l|}{$\begin{array}{l}\text { Association of urinary tract } \\
\text { reconstructive procedures }\end{array}$} \\
\hline ileal neobladder & 18 \\
\hline Bricker diversion & 34 \\
\hline Cutaneous urostomy & 25 \\
\hline Association of perineal reconstructions & 2 \\
\hline Median length of surgery $(\mathrm{min})$ & $420 \min (250-600 \mathrm{~min})$ \\
\hline Median estimated blood loss (ml) & $850 \mathrm{ml}(300-1500 \mathrm{ml})$ \\
\hline Number of transfused red blood cells packs & $2(0-4)$ \\
\hline Median length of hospital in stay (days) & 16 days (10-56 days) \\
\hline \multicolumn{2}{|l|}{ Postoperative morbidity - } \\
\hline \multicolumn{2}{|l|}{ Dindo-Clavien grade } \\
\hline $1-2$ & 12 \\
\hline $3-4$ & 22 \\
\hline Postoperative mortality - Dindo-Clavien grade 5 & 3 \\
\hline
\end{tabular}

Table III. Histopathological characteristics of the specimens after pelvic exenteration.

\begin{tabular}{lc}
\hline Characteristics & Value \\
\hline Tumoral dimension & \\
$<5 \mathrm{~cm}$ & 37 \\
$>5 \mathrm{~cm}$ & 63 \\
Type of resection & \\
R0 & 68 \\
R1 & 32 \\
Positive retrieved lymph nodes & \\
Pelvic & 56 \\
Para-aortic & 21 \\
Inguinal & 4 \\
Lympho-vascular space invasion & \\
Yes & 65 \\
No & 31 \\
Not reported & 4 \\
Peri-neural invasion & \\
Yes & 54 \\
No & 19 \\
Not reported & 27 \\
\hline
\end{tabular}


Postoperatively, 63 patients were submitted to adjuvant chemotherapy, 40 of whom were submitted to surgery for primary tumors and 23 cases submitted to surgery for relapsed lesions. At the end of the study, 25 cases were diagnosed with relapse after a median follow-up period of 18 months, 10 cases presenting local and distant metastases, eight cases presenting exclusively distant metastases and seven cases being diagnosed with local relapse. Not surprisingly, all 17 cases who were diagnosed with local recurrence presented positive resection margins at the time of pelvic exenteration. Among the 25 cases diagnosed with recurrent disease, 18 cases had been submitted to surgery for recurrent disease.

The analysis of the long-term outcomes revealed a median overall survival of 38.7 months and a median disease-free survival period of 21.3 months. In univariate analysis, the overall survival was significantly influenced by the origin of the primary tumor (rectal versus gynaecological tumor) as well as by the completeness of resection. Cases submitted to surgery for colorectal lesions experienced a median overall survival of 42 months, while cases submitted to surgery for gynaecological malignancies experienced a median overall survival of 35 months $(p=0.04)$. In the meantime, patients submitted to an R0 resection reported a median overall survival time of 46.4 months, significantly higher when compared to those in whom an $\mathrm{R} 1$ resection was performed (with a median overall survival time of 10.4 months, $p=0.001$ ). However, the dimension of the pelvic tumor did not significantly impact on survival (cases in which the lesion was higher $5 \mathrm{~cm}$ experienced an overall survival of 39.3 months while cases with lower dimensions tumor reported a median overall survival time of 36.1 months, $p=0.878$ ). The histopathological type or the degree of differentiation did not significantly influence the long-term outcomes. However, tumors presenting lymphovascular invasion were associated with a significantly poorer outcome when compared to cases without invasion of the lymphovascular space. At a mean follow-up of two years, the survival rates were $72 \%$ for primary lesions and $63 \%$ for recurrent lesions.

\section{Discussion}

Patients diagnosed with locally advanced or recurrent pelvic malignancies seem to have poor response rates to chemoirradiation. In cases where an oncological palliative treatment is performed death usually occurs in the following one or two years (6-8). Therefore, the only potential curative solution is represented by pelvic exenteration, an ultraradical procedure which might induce a significant modification of the quality of life (9-11). However, due to the paucity of other therapeutic strategies, it remains the option of choice in an important number of cases, especially if a complete resection with negative margins is to be expected. Although the surgical techniques, as well as the postoperative management of these patients has significantly improved, this type of procedure is still associated with an important risk of postoperative complications. In our study, the overall morbidity rate was $37 \%$ while the mortality within the first 30 days was $3 \%$, comparable to the rates reported by other published studies $(2,3)$. As for the longterm outcomes, studies published so far have demonstrated the efficacy of the method for both rectal and gynaecological malignancies, with an estimated five-year survival rate of 43 $77 \%$ for primary gynaecological or rectal tumors and $8-41 \%$ for recurrent lesions (12-14).

When it comes to the influence of the tumoral volume on the long-term outcomes, conflicting results have been published so far. While certain authors consider that lesions larger than $5 \mathrm{~cm}$ should not be submitted to pelvic exenteration, other authors have demonstrated that the volume itself does not influence the overall survival rate $(2,15)$. While the largest dimension of the tumors which should be amenable to pelvic exenteration is submitted to a permanent debate, it seems that almost all authors agree that the status of the resection margins play a crucial role. Therefore, most authors that have studied the long-term prognostic factors after pelvic exenteration have demonstrated that the status of the resection margins significantly influence the long-term outcomes (2, 14). It has been widely demonstrated that an R0 resection is maybe the most important factor for improved survival, such a resection being more often obtained in cases where an en bloc resection of the involved organs is feasible. However, this fact is even more difficult in patients presenting recurrent disease, as well as in cases with a heavy history of preirradiation. In such cases, an important anatomical distortion of the tissues is usually encountered. One of the most difficult tasks when performing surgery in such cases is to distinguish between the tumoral invasion, the radiationinduced fibrosis or local inflammation (16). In such cases, according to Hockel's study, there is a high risk of performing an intralesional resection, which won't provide any significant benefit in regard to the long-term outcomes of the patient (17, 18). In order to minimise the number of these situations, Hockel et al. proposed the concept of laterally extended endopelvic resections with satisfactory results for both locally invasive and relapsed gynaecological malignancies. According to this theory, the central pelvic tumor should be removed en bloc with the internal iliac vessel system, which enables the surgeon to perform a complete excision of the urogenital mesentery, the pelvic muscles and the regional lymph node groups (18). In our study, laterally extended endopelvic resections were performed in five cases diagnosed with relapsed cervical cancer, where an $\mathrm{R} 0$ resection with negative margins was achieved in all cases.

As expected, the long-term follow-up in our study demonstrated a higher disease-free survival for patients 
submitted to surgery for primary lesions when compared to those treated for recurrent disease. However, published studies have demonstrated that patients submitted to surgery for relapsed rectal cancer will experience better overall survival when compared to other primaries $(14,19)$. One of the largest studies which investigated this subject was the one conducted by Sagar et al., who reported a disease-free survival rate of $60 \%$ at a 25 months follow-up in cases submitted to surgery for pelvic exenteration for relapsed rectal cancer (20). Similarly to our study, other authors have also observed that the rate of relapse after pelvic exenteration is higher in patients submitted to surgery for recurrent disease (14).

An interesting study conducted on pelvic exenteration for locally advanced pelvic tumors was published by Kuhrt et $a l$. in 2012 (21). The study included 36 patients submitted to surgery for colorectal lesions and 17 cases submitted to surgery for non-colorectal lesions and demonstrated that colorectal primaries had a significantly improved survival. This aspect was explained by the fact that the rates of R0 resection were higher among patients with colorectal tumors. In the meantime, patients submitted to pelvic exenteration for colorectal tumors in which a $\mathrm{R} 0$ resection was feasible experienced a significantly better outcome when compared to those in which the origin was also colorectal but in whom an R1 or R2 resection was achievable. Interestingly, among patients with colorectal cancer there was no difference in terms of survival between patients submitted to surgery for locally advanced or relapsed lesions (21).

\section{Conclusion}

Pelvic exenteration remains a demanding surgical procedure which still associates a high risk of postoperative complications and therefore it should be performed in selected cases. However, a significant survival benefit is expected when negative resection margins can be achieved. Other prognostic factors include the origin of the primary tumor (colorectal versus non-colorectal primaries), as well as the pathological findings, such as the presence of lymphovascular invasion.

\section{Conflicts of Interest}

The Authors do not have any conflicts of interest to declare regarding this study.

\section{Authors' Contributions}

NB - Performed surgical procedures; IB - prepared the manuscript; $\mathrm{AN}, \mathrm{SD}, \mathrm{AC}$ - data analysis, prepared the tables; MV, IB - part of the surgical team, IB - adviced about surgical oncology procedure, revised the final draft of the manuscript.

\section{Acknowledgements}

This work was supported by the project entitled „Multidisciplinary Consortium for Supporting the Research Skills in Diagnosing, Treating and Identifying Predictive Factors of Malignant Gynecologic Disorders", project number PN-III-P1-1.2-PCCDI2017-0833.

\section{References}

1 Brunschwig A: Complete excision of pelvic viscera for advanced carcinoma; a one-stage abdominoperineal operation with end colostomy and bilateral ureteral implantation into the colon above the colostomy. Cancer 1: 177-183, 1948. PMID: 18875031. DOI: 10.1002/1097-0142(194807)1:2<177::aid-cncr 2820010203>3.0.co;2-a

2 de Gregorio N, de Gregorio A, Ebner F, Friedl TWP, Huober J, Hefty R, Wittau M, Janni W and Widschwendter P: Pelvic exenteration as ultimate ratio for gynecologic cancers: singlecenter analyses of 37 cases. Arch Gynecol Obstet 300: 161-168, 2019. PMID: 31011878. DOI: 10.1007/s00404-019-05154-4

3 Hockel $M$ and Dornhofer $\mathrm{N}$ : Pelvic exenteration for gynaecological tumours: achievements and unanswered questions. Lancet Oncol 7: 837-847, 2006. PMID: 17012046. DOI: $10.1016 / \mathrm{S} 1470-2045(06) 70903-2$

4 Hockel M: Ultra-radical compartmentalized surgery in gynaecological oncology. Eur J Surg Oncol 32: 859-865, 2006. PMID: 16690243. DOI: 10.1016/j.ejso.2006.03.040.

5 Clavien PA, Barkun J, de Oliveira ML, Vauthey JN, Dindo D, Schulick RD, de Santibanes E, Pekolj J, Slankamenac K, Bassi C, Graf R, Vonlanthen R, Padbury R, Cameron JL and Makuuchi M: The Clavien-Dindo classification of surgical complications: five-year experience. Ann Surg 250: 187-196, 2009. PMID: 19638912. DOI: 10.1097/SLA.0b013e3181b13ca2.

6 Wong CS, Cummings BJ, Brierley JD, Catton CN, McLean M, Catton $\mathrm{P}$ and Hao Y: Treatment of locally recurrent rectal carcinoma - results and prognostic factors. Int J Radiat Oncol Biol Phys 40: 427-435, 1998. PMID: 9457832. DOI: 10.1016/ s0360-3016(97)00737-2.

7 Sugiyama T, Yakushiji M, Noda K, Ikeda M, Kudoh R, Yajima A, Tomoda Y, Terashima Y, Takeuchi S, Hiura M, Saji F, Takahashi T, Umesaki N, Sato S, Hatae M and Ohashi Y: Phase II study of irinotecan and cisplatin as first-line chemotherapy in advanced or recurrent cervical cancer. Oncology 58: 31-37, 2000. PMID: 10644938. DOI: 10.1159/000012076.

8 Gerard JP, Romestaing P and Chapet O: Radiotherapy alone in the curative treatment of rectal carcinoma. Lancet Oncol 4: 158166, 2003. PMID: 12623361.

9 Bacalbasa N, Balescu I and Filipescu A: Debulking surgery for high-grade serous endometrial cancer with disseminated peritoneal lesions. In Vivo 31: 719-722, 2017. PMID: 28652446. DOI: 10.21873 /invivo. 11120

10 Bacalbasa N, Balescu I and Tomescu D: Partial cystectomy with distal ureteral resection and re-implantation for locally invasive cervical cancer. Anticancer Res 35: 5539-5542, 2015. PMID: 26408722.

11 Bacalbasa N, Tomescu D and Balescu I: Use of inflated foley catheters to prevent early empty pelvis complications following pelvic exenteration. Anticancer Res 35: 5543-5546, 2015. PMID: 26408723. 
12 Goldberg GL, Sukumvanich P, Einstein MH, Smith HO, Anderson PS and Fields AL: Total pelvic exenteration: The Albert Einstein College of Medicine/Montefiore Medical Center Experience (1987 to 2003). Gynecol Oncol 101: 261-268, 2006. PMID: 16426668. DOI: 10.1016/j.ygyno.2005.10.011

13 Ike H, Shimada H, Ohki S, Yamaguchi S, Ichikawa Y and Fujii $\mathrm{S}$ : Outcome of total pelvic exenteration for locally recurrent rectal cancer. Hepatogastroenterology 50: 700-703, 2003. PMID: 12828064

14 Zoucas E, Frederiksen S, Lydrup ML, Mansson W, Gustafson P and Alberius P: Pelvic exenteration for advanced and recurrent malignancy. World J Surg 34: 2177-2184, 2010. PMID: 20512493. DOI: $10.1007 / \mathrm{s} 00268-010-0637-7$

15 Höckel M: Pelvic recurrences of cervical cancer. Relapse pattern, prognostic factors and the role of extended radical treatment. J Pelvic Surg 5: 255-266, 1999.

16 Rodel C, Grabenbauer GG, Matzel KE, Schick C, Fietkau R, Papadopoulos T, Martus P, Hohenberger W and Sauer R: Extensive surgery after high-dose preoperative chemoradiotherapy for locally advanced recurrent rectal cancer. Dis Colon Rectum 43: 312-319, 2000. PMID: 10733111. DOI: 10.1007/ bf02258294

17 Jurado M, Alcazar JL and Martinez-Monge R: Resectability rates of previously irradiated recurrent cervical cancer (PIRCC) treated with pelvic exenteration: is still the clinical involvement of the pelvis wall a real contraindication? a twenty-year experience. Gynecol Oncol 116: 38-43, 2010. PMID: 19878978. DOI: $10.1016 /$ j.ygyno.2009.09.035
18 Hockel M: Long-term experience with (laterally) extended endopelvic resection (LEER) in relapsed pelvic malignancies. Curr Oncol Rep 17: 435, 2015. PMID: 25687807. DOI: 10.1007/ s11912-014-0435-8

19 Pawlik TM, Skibber JM and Rodriguez-Bigas MA: Pelvic exenteration for advanced pelvic malignancies. Ann Surg Oncol 13: 612-623, 2006. PMID: 16538402. DOI: 10.1245/ASO. 2006.03.082

20 Sagar PM, Gonsalves S, Heath RM, Phillips N and Chalmers AG: Composite abdominosacral resection for recurrent rectal cancer. Br J Surg 96: 191-196, 2009. PMID: 19160364. DOI: 10.1002/bjs.6464

21 Kuhrt MP, Chokshi RJ, Arrese D and Martin EW Jr.: Retrospective review of pelvic malignancies undergoing total pelvic exenteration. World J Surg Oncol 10: 110, 2012. PMID: 22703863. DOI: $10.1186 / 1477-7819-10-110$
Received August 12, 2019

Revised September 1, 2019

Accepted September 3, 2019 